\title{
HMGB1 and thrombin mediate the blood-brain barrier dysfunction acting as biomarkers of neuroinflammation and progression to neurodegeneration in Alzheimer's disease
}

\author{
Barry W. Festoff ${ }^{1,2}$, Ravi K. Sajja ${ }^{3}$, Patrick van Dreden ${ }^{4}$ and Luca Cucullo ${ }^{3 *}$ (i)
}

\begin{abstract}
Background: The blood-brain barrier (BBB) dysfunction represents an early feature of Alzheimer's disease (AD) that precedes the hallmarks of amyloid beta (amyloid $\beta$ ) plaque deposition and neuronal neurofibrillary tangle (NFT) formation. A damaged BBB correlates directly with neuroinflammation involving microglial activation and reactive astrogliosis, which is associated with increased expression and/or release of high-mobility group box protein 1 (HMGB1) and thrombin. However, the link between the presence of these molecules, BBB damage, and progression to neurodegeneration in AD is still elusive. Therefore, we aimed to profile and validate non-invasive clinical biomarkers of BBB dysfunction and neuroinflammation to assess the progression to neurodegeneration in mild cognitive impairment (MCl) and AD patients.
\end{abstract}

Methods: We determined the serum levels of various proinflammatory damage-associated molecules in aged control subjects and patients with $\mathrm{MCl}$ or $\mathrm{AD}$ using validated ELISA kits. We then assessed the specific and direct effects of such molecules on BBB integrity in vitro using human primary brain microvascular endothelial cells or a cell line.

Results: We observed a significant increase in serum HMGB1 and soluble receptor for advanced glycation end products (SRAGE) that correlated well with amyloid beta levels in AD patients (vs. control subjects). Interestingly, serum HMGB1 levels were significantly elevated in $\mathrm{MCl}$ patients compared to controls or AD patients. In addition, as a marker of BBB damage, soluble thrombomodulin (sTM) antigen, and activity were significantly (and distinctly) increased in MCI and $A D$ patients. Direct in vitro BBB integrity assessment further revealed a significant and concentration-dependent increase in paracellular permeability to dextrans by HMGB1 or a-thrombin, possibly through disruption of zona occludins-1 bands. Pre-treatment with anti-HMGB1 monoclonal antibody blocked HMGB1 effects and leaving BBB integrity intact.

Conclusions: Our current studies indicate that thrombin and HMGB1 are causal proximate proinflammatory mediators of BBB dysfunction, while sTM levels may indicate BBB endothelial damage; HMGB1 and sRAGE might serve as clinical biomarkers for progression and/or therapeutic efficacy along the AD spectrum.

Keywords: Biomarkers, Blood-brain barrier, Clinical, DAMPS, HMGB1, Neuroinflammation, Neurodegeneration, Permeability, Thrombin

\footnotetext{
* Correspondence: luca.cucullo@ttuhsc.edu

${ }^{3}$ Department of Pharmaceutical Sciences, Texas Tech University Health

Sciences Center, 1300 S. Coulter Street, Amarillo, TX 79106, USA

Full list of author information is available at the end of the article
} 


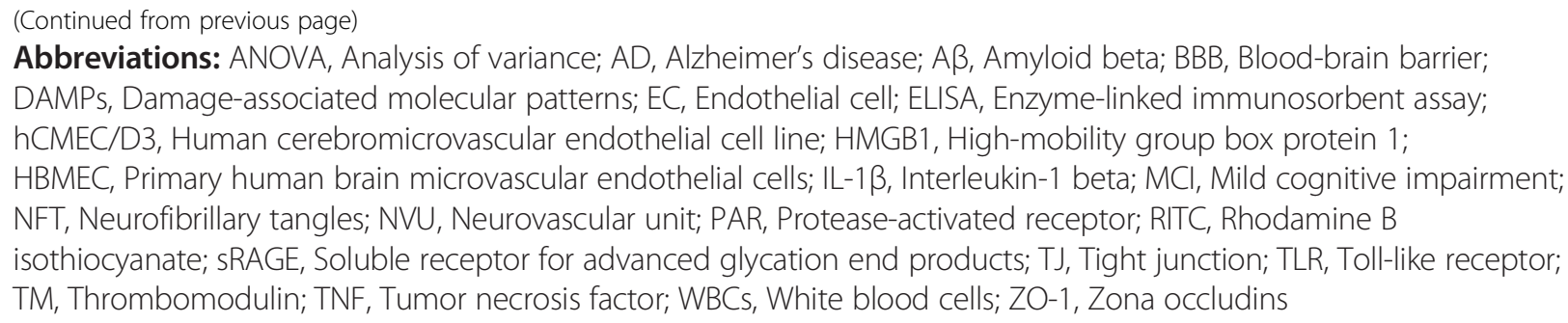

\section{Background}

Despite overarching evidence, the amyloid hypothesis in Alzheimer's disease (AD) first elaborated in 1991, has yet to provide positive outcomes notwithstanding the billions spent on clinical trials [1]. The neuropathological hallmarks of AD are extracellular amyloid beta $(\mathrm{A} \beta) /$ neuritic plaques and intracellular neurofibrillary tangle (NFT) formation [2]. In association with these hallmarks, soluble $A \beta$ levels increase in the blood, both in $\mathrm{AD}$ patients and transgenic mouse models [3-5], while the brain amyloid $\beta$ aggregates promote a neuroinflammatory response mediated by activated microglia, astrocytes, and microvascular endothelial cells (ECs) $[6,7]$. Since late-onset AD (LOAD) is not associated with such manifestations information from $\mathrm{AD}$, transgenic animal models cannot be fully extrapolated to human AD pathology. Furthermore, microglial activation and other aspects of neuroinflammation involving oxidative stress (reactive oxygen species (ROS), nitric oxide (NO)) actually precede neuronal damage $[8,9]$, prior to AD histopathologic lesions. Moreover, a critical characteristic of neuroinflammation is the disruption of the blood-brain barrier (BBB) that extends beyond the tissue or cellular pathophysiology of endothelial cell (EC) dysfunction to the neurovascular unit (NVU), including astrocytic end-feet, microglia, neurons, and pericytes [10-12]. Recent National Institutes of Health (NIH) workshops have emphasized key areas that must be focused on as it relates to AD neuroinflammation research involving the BBB: (1) transport of $A \beta$ and other macromolecules in and out of the brain, i.e., permeability; (2) BBB as both the source and target of inflammatory factors; and (3) effects of oxidative stress, ROS, and NO on BBB. Besides astrogliosis, activation and transmigration of blood-borne substances and circulating immune cells into the CNS is a less studied and underappreciated area in AD research [13-16]. The precise molecular factors governing the initial $\mathrm{BBB}$ damage leading to neurodegeneration, in general, and AD, in particular, are not well understood.

Thrombin and high-mobility group box protein 1 (HMGB1) are key molecules of two most potent host defense systems that converge on the innate immune system, coagulation, and inflammation. We postulated that they may play significant roles in the BBB disruption since both are proinflammatory and both are known to disrupt vascular barriers in other tissues [17-20]. Thrombin is a proinflammatory serine protease that is well known for its essential role as the ultimate protease in the coagulation pathway. HMGB1 is a non-histone nuclear protein with dual functions depending on localization. Within the cells, it is localized primarily to the nucleus where it binds DNA and plays a role in transcriptional regulation [21]. However, extracellular HMGB1 serves as a proinflammatory cytokine and is a late mediator of sepsis [22]. Beyond infections, HMGB1 has pathogenic roles during trauma and sterile inflammation, such as systemic inflammatory response syndrome (SIRS), where elevated levels in sera orchestrate key events including leukocyte recruitment and white blood cell (WBC) induction to secrete inflammatory cytokines [23, 24].

Relevant to our studies, HMGB1 impairs memory behavior in mice that is mediated via Toll-like receptor 4 (TLR4) and the receptor for advanced end product glycation (RAGE) [25]. These pre-clinical data correlate with clinical studies showing that sepsis survivors have permanent cognitive deficits [26] and that these may also be mediated via HMGB1, but the precise mechanism remains unknown. HMGB1 and another alarmin, $\mathrm{S} 100 \mathrm{~B}$, along with $\mathrm{A} \beta$, are now considered as three significant damage or danger-associated molecular patterns (DAMPs) that "fan the flame" [27] of neuroinflammation in AD [28]. How they might do this is currently unknown. As an approach to this problem, we first measured levels of these DAMPs in mild cognitive impairment (MCI), AD, and normal aged subjects and then used pure proteins in the range of these levels to perturb human BBB function in vitro.

\section{Methods}

\section{Human subjects and specimen collection}

The human study was approved by the institutional review board at the University of Kansas Medical Center (KUMC) and was performed in compliance with the Helsinki Declaration. All subjects were recruited from the KU Alzheimer Disease Center (ADC) following a written informed consent, and all demographic information was de-identified. Subjects in the AD, MCI, and 
aged control cohorts met the criteria as outlined in the 2011-2012 NIA/Alzheimer's Association Guidelines for AD by McKhann et al. [29] and for MCI by Albert et al. [30]. Furthermore, all subjects were screened by clinical dementia rating (CDR) and neuropsychological testing as defined by the Uniform Data Set of the Alzheimer Disease Centers and underwent a consensus review. $\mathrm{MCI}$ patients were further classified as "MCI due to AD" if another more likely cause was not identified. Our MCI cohort patients were all designated MCI due to AD.

Venous blood collection was performed with BD Vaccutainer blood collection tubes and centrifuged. The serum samples were aliquoted and stored at $-80^{\circ} \mathrm{C}$ until analysis. Demographic information for these patient groups is shown in Table 1.

\section{Cell culture}

The human cerebromicrovascular endothelial cell line (hCMEC/D3) was a gift from Dr. P.O. Couraud (INSERM, France). This cell line has been widely used as a representative model of human BBB in vitro for mechanistic studies involving the molecular regulation of BBB integrity [31]. Cells between passages 28 and 31 were cultured in HEPES-buffered EBM-2 media supplemented with growth factors and antibiotics and maintained at $37{ }^{\circ} \mathrm{C}$ with $5 \% \mathrm{CO}_{2}$ exposure. In preliminary experiments, primary human brain microvascular endothelial cells (HBMEC) were cultured in Dr. KY Kim's laboratory (KUMC) as described previously [32].

\section{Analysis of serum biomarkers}

Human serum samples were thawed on ice and analyzed by enzyme-linked immunosorbent assay (ELISA) for quantification of HMGB1 (Novatein Biosciences, Woburn, MA), s100 $\beta$ (EMD Millipore, Billerica, MA), amyloid $\beta$ (aa 1-42), and soluble receptor for advanced glycation end products (sRAGE) from R\&D Systems (Minneapolis, MN, USA), according to the manufacturers' instructions. Soluble thrombomodulin (sTM) antigen (TMa) levels were assayed by kit (Stago, Gennevilliers, France), and sTM activity was measured as described previously [33].

\section{BBB integrity assessment}

Cells were seeded on Corning Transwell $^{\circ}$ polyester membrane inserts (12-well, $0.4 \mu \mathrm{m}$ pore size) as previously described [31]. Monolayer integrity and morphology were periodically assessed by transendothelial electrical resistance (TEER; $\Omega \mathrm{cm}^{2}$ ) and phase contrast microscopy, respectively. Cells were maintained in treatment media (EMB-2 supplemented with $10 \mathrm{mM}$ HEPES, antibiotics, and $1 \%$ human serum) overnight prior to treatment with human recombinant HMGB1 protein (rHMGB1) with endotoxin levels lower than 0.1 EU per microgram of the protein $(R \& D$ Systems, Minneapolis,
Table 1 Human subject demographics (gender and age were shown for each subject in $\mathrm{AD}, \mathrm{MCl}$, and control cohorts at the time of blood collection)

\begin{tabular}{|c|c|c|c|}
\hline Patient ID & Gender & Age at draw & Dx at draw \\
\hline 1 & $\mathrm{~F}$ & 80 & $A D$ \\
\hline 2 & F & 77 & $A D$ \\
\hline 3 & F & 81 & $A D$ \\
\hline 4 & $F$ & 60 & $A D$ \\
\hline 5 & M & 63 & $A D$ \\
\hline 6 & M & 76 & $A D$ \\
\hline 7 & M & 73 & $A D$ \\
\hline 8 & M & 81 & $A D$ \\
\hline 9 & M & 67 & $A D$ \\
\hline 10 & M & 70 & $A D$ \\
\hline 11 & M & 84 & $A D$ \\
\hline 12 & M & 88 & $A D$ \\
\hline 1 & F & 67 & $\mathrm{MCl}$ \\
\hline 2 & M & 88 & $\mathrm{MCl}$ \\
\hline 3 & M & 76 & $\mathrm{MCl}$ \\
\hline 4 & M & 74 & $\mathrm{MCl}$ \\
\hline 5 & M & 77 & $\mathrm{MCl}$ \\
\hline 6 & M & 64 & $\mathrm{MCl}$ \\
\hline 7 & M & 72 & $\mathrm{MCl}$ \\
\hline 8 & M & 84 & $\mathrm{MCl}$ \\
\hline 9 & M & 68 & $\mathrm{MCl}$ \\
\hline 10 & M & 72 & $\mathrm{MCl}$ \\
\hline 11 & M & 75 & $\mathrm{MCl}$ \\
\hline 12 & M & 65 & $\mathrm{MCl}$ \\
\hline 1 & $\mathrm{~F}$ & 77 & $\mathrm{NL}$ \\
\hline 2 & F & 76 & $\mathrm{NL}$ \\
\hline 3 & F & 78 & $\mathrm{NL}$ \\
\hline 4 & F & 66 & $\mathrm{NL}$ \\
\hline 5 & $F$ & 69 & $\mathrm{NL}$ \\
\hline 6 & F & 74 & $\mathrm{NL}$ \\
\hline 7 & F & 81 & $\mathrm{NL}$ \\
\hline 8 & F & 75 & $\mathrm{NL}$ \\
\hline 9 & $\mathrm{~F}$ & 72 & $\mathrm{NL}$ \\
\hline 10 & F & 66 & $\mathrm{NL}$ \\
\hline 11 & M & 69 & $\mathrm{NL}$ \\
\hline 12 & M & 76 & $\mathrm{NL}$ \\
\hline
\end{tabular}

MN, USA). Following $3 \mathrm{~h}$ of treatment with rHMGB1 $(5,10$, and $50 \mathrm{ng} / \mathrm{mL}), \alpha$-thrombin $(0.5,1$, and $5 \mathrm{nM})$ or control media, inserts were transferred to new 12-well plates and transport buffer added (pre-warmed HBSS buffer with antibiotics and $0.5 \% \mathrm{BSA}$ ) to the apical $(500 \mu \mathrm{L})$ and basal compartment $(1000 \mu \mathrm{L})$. After incubation for $20 \mathrm{~min}$ at $37{ }^{\circ} \mathrm{C}$ under $5 \% \mathrm{CO}_{2}$ exposure, a 
mixture of florescent dextrans of increasing molecular sizes (FITC-4 kDa, $10 \mathrm{mg} / \mathrm{mL}$; Cascade Blue $10 \mathrm{kDa}$, $5 \mathrm{mg} / \mathrm{mL}$; and rhodamine $\mathrm{B}$ isothiocyanate (RITC)$70 \mathrm{kDa}, 10 \mathrm{mg} / \mathrm{mL}$ ) was added to the apical compartment. Dextran permeability (luminal to abluminal flux) was measured at 30 min following the addition of dextrans. Apparent permeability coefficients (Pe; centimeters per second) were calculated and expressed as percent control [31]. For pharmacological inhibition (validation) studies, cells were co-incubated with anti-HMGB1 antibody (gift from Dr. Michael Bustin, NIH) or non-specific control antibody for the indicated duration.

\section{Immunofluorescence analysis}

Immunocytochemistry (ICH) for zona occludin-1 (ZO-1) was performed using primary anti-ZO-1 antibody (Zymed Laboratories, San Francisco, CA), Alexa Fluor 488-conjugated secondary antibody and 4,6-diamidino-2-phenylindole (DAPI; Molecular Probes, Eugene, OR) as described in [32].

\section{Statistical analysis}

Data were expressed as mean \pm SEM or SD. Data were analyzed by one-way analysis of variance (ANOVA) followed by Dunnett's or Tukey's post hoc multiple comparison tests using GraphPad Prism Software Inc. (La Jolla, CA, USA). $P$ value was set to less than 0.05 for statistical significance.

\section{Results}

Serum profile of proinflammatory "damage-associated" factors in $A D, M C l$, and age-matched normal subjects

Part of the criteria for $\mathrm{AD}$ and $\mathrm{MCI}$ due to $\mathrm{AD}$ infers that other more likely diagnoses are less likely than AD. Although it does not totally eliminate, it clearly should limit contamination by other diagnoses such as vascular dementia, Parkinson's disease with dementia, and primary systemic inflammatory diseases.

Levels of various proinflammatory markers including $A \beta$, sRAGE, HMGB1, and $S 100 \beta$ in serum samples derived from control subjects $(\mathrm{NL}), \mathrm{AD}$, or $\mathrm{MCI}$ patients (see Table 1 for demographic information) were measured by ELISA. As shown in Fig. 1, A $\beta$ levels were significantly higher in $\mathrm{AD}$ patients when compared to $\mathrm{MCI}$ and NL. No statistically significant differences were noted between MCI and NL groups. An unprecedented pathophysiological parallelism between $A \beta$ and sRAGE (the soluble, circulating form of RAGE) [34, 35] levels was also noted, as sRAGE was equally increased in the plasma of AD patients vs. MCI and controls (Fig. 1). In contrast, parallel measurements of HMGB1 in the MCI cohort was significantly higher than the levels measured in $\mathrm{AD}$ and control subjects. A side-by-side comparison between $\mathrm{AD}$ and NL revealed a higher trend for HMGB1 levels in $\mathrm{AD}$ patients although the results were not statistically significant. No significant differences between the three groups were observed with respect to $S 100 \beta$ levels.

The critical message from these results is that these proinflammatory damage-associated molecules [28] (with the exception of s100 $\beta$ ) show specific patterns of expression in relationship to the continuum from normal aging $\rightarrow$ M$\mathrm{CI} \rightarrow \mathrm{AD}$. This strongly suggests that HMGB1 and/or sRAGE in addition to $A \beta$ could have a potential use as prognostic/diagnostic markers for $\mathrm{AD}$ and $\mathrm{MCI}$.

Serum expression/activity of soluble thrombomodulin in $\mathrm{AD}, \mathrm{MCl}$, and control subjects

Additionally, in our efforts to develop a validated, noninvasive biomarker for $\mathrm{BBB}$ EC damage in $\mathrm{AD}$, we measured both sTM antigen (TM-Ag) and TM activity (TMa; which converts protein $\mathrm{C}$ to activated $\mathrm{PC}$; see "Methods" section). As shown in Fig. 2, we found significant increase in TMa above age-matched controls when $\mathrm{MCI}$ and $\mathrm{AD}$ levels were grouped (upper panel). We also observed that MCI levels were, in fact, statistically higher than those measured in $\mathrm{AD}$ patients (middle panel). However, as shown in Fig. 2 (bottom panel), the following relationship was found for TM Ag: $\mathrm{AD}>\mathrm{MCI}>-$ control (lower panel) with a significant increase in $\mathrm{AD}$ vs. control subjects. Similar to remitting relapsing multiple sclerosis (RRMS) patients [36], increased levels of sTM occur in sera of AD and MCI patients as compared with controls (Fig. 2).

\section{Effects of proinflammatory HMGB1 and thrombin on BBB permeability in vitro}

To directly assess microvascular damage associated with elevated levels of these proinflammatory DAMPs in AD and MCI patients, we next determined whether HMGB1 and/or the proinflammatory coagulation protease, thrombin, players in vascular barrier dysfunction in other tissues could disrupt BBB integrity. As shown in Fig. 3a, one-way ANOVA revealed that pre-treatment with recombinant human HMGB1 $(10 \mathrm{ng} / \mathrm{mL})$ protein for 3 or $6 \mathrm{~h}$ significantly increased BBB apical to basal permeability to FITC dextran across the HBMEC monolayers ( $p<0.001$ vs. control). We also observed that the magnitude of dextran flux was more pronounced with the duration of exposure to HMGB1 ( $p<0.01,6$ vs. 3 h; see inset in Fig. 3a). Importantly, HMGB1-induced increase in BBB paracellular dextran permeability could be significantly and specifically blocked by neutralizing antibody against HMGB1 [37], whereas control (pre-immune) antibody failed to suppress HMGB-1-induced BBB disruption at either incubation time (Fig. 3a). Intriguingly, our preliminary data also indicated that a specific fragment of rTM, called TM-D1, the C-type lectin-like domain [38], can effectively block 

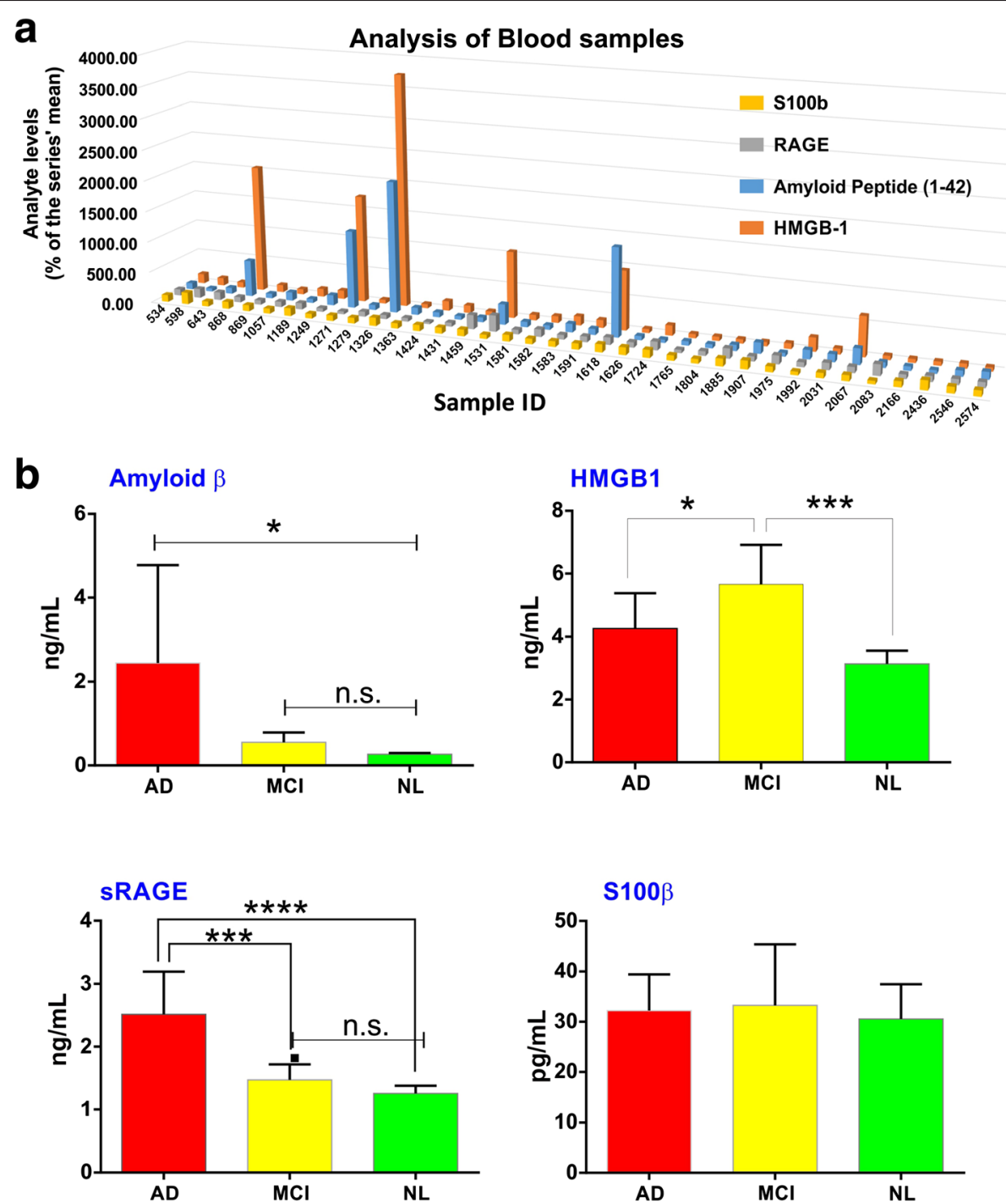

Fig. 1 Serum profiling of clinical biomarkers of neuroinflammation to predict the conversion (continuum) of $M C l$ to $A D$. a The serum levels of $A \beta$, HMGB1, sRAGE, and s100ß are shown individually for each subject belonging to age-matched control (NL), MCl, or AD cohorts to facilitate the correlation of these markers. b Serum levels of each DAMP expressed as mean $\left( \pm\right.$ SD) for each cohort. ${ }^{*} p<0.05$ and ${ }^{* * *} p<0.01$. n.s. not significant

rHMGB1 activity, thus protecting the $\mathrm{BBB}$ against this DAMP (data not shown).

Similarly, when we tested the effects of thrombin on BBB integrity, we observed a significant and concentrationdependent increase in FITC-dextran permeability across the HBMEC monolayers (Fig. 3b). For instance, higher thrombin concentrations $(5 \mathrm{nM})$ more potently induced BBB disruption $(p<0.0001$ vs. control) when compared to dextran permeability resulting from exposure to 0.5 or $1 \mathrm{nM}(p<0.001)$. However, we did not observe a further elevation in thrombin-induced BBB dextran flux with longer exposure time ( 3 vs. 6 h), suggesting a maximum and sustained response following $3 \mathrm{~h}$ of exposure. The negative impact of HMGB1 and thrombin on BBB integrity is further confirmed by corresponding immunostaining of
HBMECs that demonstrated a significant downregulation of ZO-1 expression at intercellular tight junctions (TJs) (Fig. 3c).

We next determined the extent of $\mathrm{BBB}$ disruption following exposure to HMGB1 by assessing the permeability to florescent dextrans of increasing molecular sizes (4-70 kDa) across the hCMEC/D3 monolayers (see "Methods" section). As shown in Fig. 4, HMGB1 exposure for $3 \mathrm{~h}$ significantly and dose-dependently increased the luminal to abluminal flux of all labeled dextrans across the hCMEC/D3 monolayers in a size-selective manner. The main effect of the treatment on 4-, 10-, and $70-\mathrm{kDa}$ dextran permeability was significant, as indicated by one-way ANOVA. Further analyses by post hoc tests revealed that high concentrations of HMGB1 

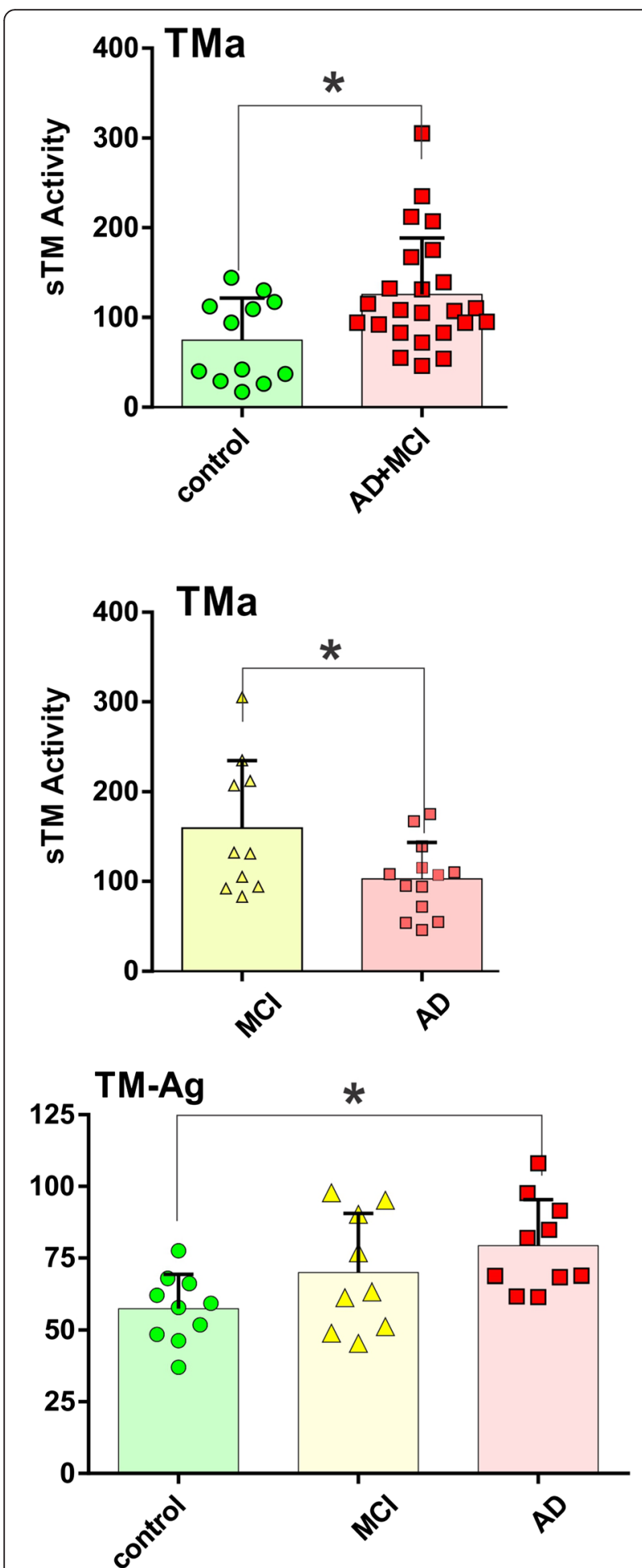

Fig. 2 Soluble thrombomodulin activity (TMa) and expression (TM-Ag) in $\mathrm{AD}, \mathrm{MCl}$, and control subject sera. TMa and TM-Ag were assayed by kits as described in the "Methods" section. Top panel represents the combined TMa in $\mathrm{MCl}$ and $\mathrm{AD}$ patients above age-matched controls. Middle panel shows the differences in TMa between $\mathrm{MCl}$ and $\mathrm{AD}$ patients. Bottom panel represents the serum levels of TM-Ag in control, $\mathrm{MCl}$, and AD subjects. Data were expressed as mean \pm SEM and ${ }^{*} p<0.05$
(10 and $50 \mathrm{ng} / \mathrm{mL}$ ) significantly increased apical to basal permeability of each dextran by 2.0-2.5-fold, as compared to aged control group $(p<0.05$ and $p<0.05$, respectively; Fig. 4). Although, HMGB1 at low concentration (5 ng/ $\mathrm{mL}$ ) showed a marked increase in BBB permeability to all dextrans, this effect was non-significant. In addition, we observed a ceiling effect on BBB permeability to 10 and $70 \mathrm{kDa}$ dextrans at HMGB1 (10 ng/ml; Fig. 4), as further increase in concentration to $50 \mathrm{ng} / \mathrm{mL}$ did not elevate the permeability to these dextrans.

\section{Discussion}

Although rigorous methods are used by the KU ADC to enroll subjects in $\mathrm{AD}, \mathrm{MCI}, \mathrm{MCI}$ due to $\mathrm{AD}$ and normal cohorts, one can never fully eliminate other systemic illnesses that escaped notice. Such recruitment methods are part of the approach that our colleagues at the KU ADC take, quite similar to methods taken at other institutional ADCs. In this regard, our current results indicate that three potent proinflammatory and damage/ danger-associated macromolecules are elevated in $\mathrm{MCI}$ and $\mathrm{AD}$ sera: $\mathrm{A} \beta$, sRAGE, and HMGB1. They further suggest that one or more may herald the conversion of $\mathrm{MCI}$ to AD (Fig. 1). Furthermore, our BBB in vitro data show that one of these, HMGB1, along with the proinflammatory coagulation protease, thrombin, are proximate mediators of BBB dysfunction. With our present results, we conclude that HMGB1 might be a better clinical biomarker, along with sRAGE and $A \beta$, to predict the course of neurodegeneration in $\mathrm{AD}$ subjects. In contrast, although it also activates RAGE [39], from our results, S100B does not appear to have applicability as a diagnostic or prognostic marker of neurodegeneration in $\mathrm{MCI}$ and $\mathrm{AD}$ patients.

Considerable evidence indicates that blood levels of sTM and von Willebrand factor (vWf) can serve as surrogate biomarkers for diffuse microvascular damage [39]. We had previously found that sTM plasma levels were higher in RRMS patients than in patients with systemic lupus erythematosus with diffuse microvascular inflammation, indicating its potential utility as a biomarker for BBB damage [36]. Relevant to our current results, others had suggested that sTM, along with vWf, might be a good biomarker for AD-related microvascular damage [40]. In our present studies, soluble forms of the anticoagulant/anti-inflammatory TM $[38,41]$ might also serve as a biomarker for microvascular damage of the $\mathrm{BBB}$ in $\mathrm{AD}$. This is strongly suggested by the pattern of expression for the sTM antigen which progressively increases from controls to $\uparrow \mathrm{MCI}$ to $\uparrow \mathrm{AD}$ although only the difference between control and $\mathrm{AD}$ was statistically significant at this stage. Perhaps future studies involving a larger cohort of patients will be able to provide additional statistical power to the analysis. When data were 
a

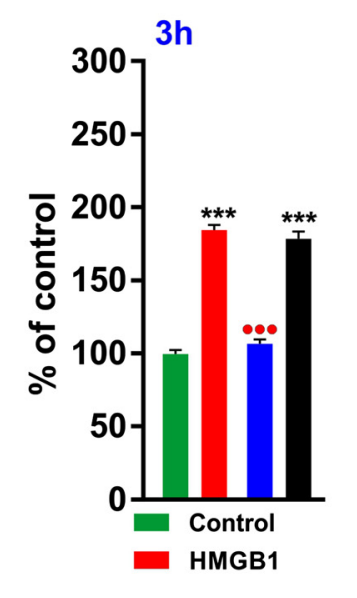

b

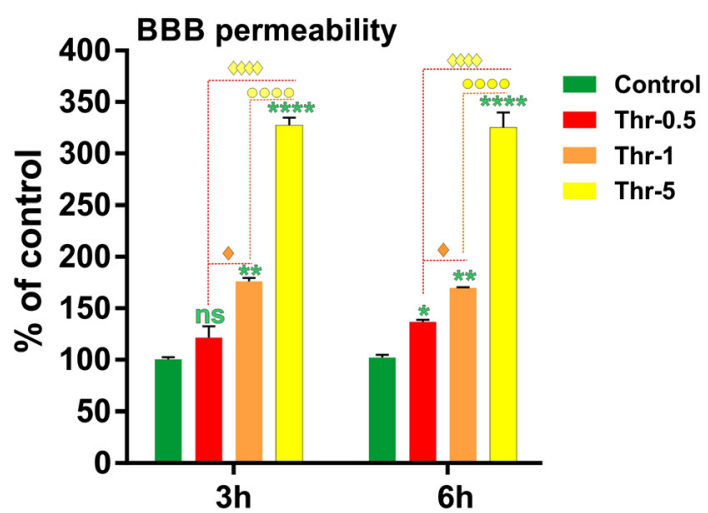

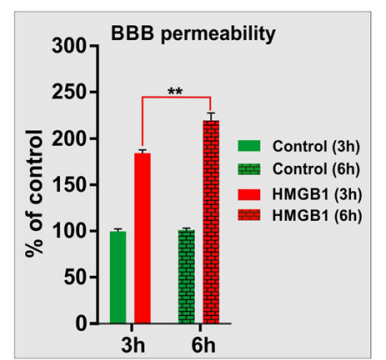

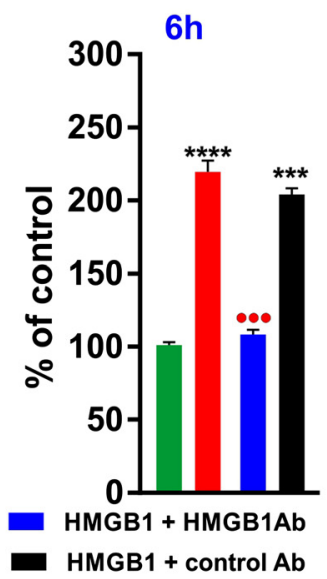

C

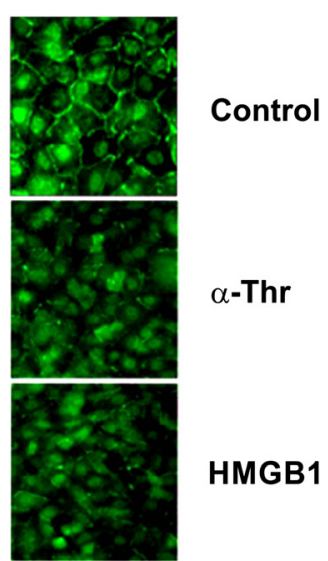

Fig. $3 \mathrm{HMGB} 1$ and thrombin impair BBB integrity. HBMEC monolayers were incubated with HMGB1 (10 ng/ml) and thrombin (Thr, $0.5,1$, or $5 \mathrm{nM})$ for 3 or $6 \mathrm{~h}$, and BBB integrity was assessed by paracellular permeability to FITC-dextran. a HMGB1 increases luminal to abluminal dextran flux across HBMECs that was reversed by anti-HMGB1 antibody (HMGB1 Ab, $10 \mathrm{ng} / \mathrm{ml}$ ). The time-dependent increase in HMGB1-induced BBB permeability was shown in the inset. $\mathbf{b}$ Exposure to thrombin ( 3 or $6 \mathrm{~h}$ ) dose-dependently increases BBB permeability to dextran in HBMECs. c HMGB1 and thrombin disrupt ZO-1 linearity at cell-cell junctions. ${ }^{*} p<0.05,{ }^{* *} p<0.01$, and ${ }^{* * *} p<0.001$ vs. control; $\bullet \bullet p<0.01$ vs. HMGB1 or HMGB1 + control Ab. $N=5 /$ condition

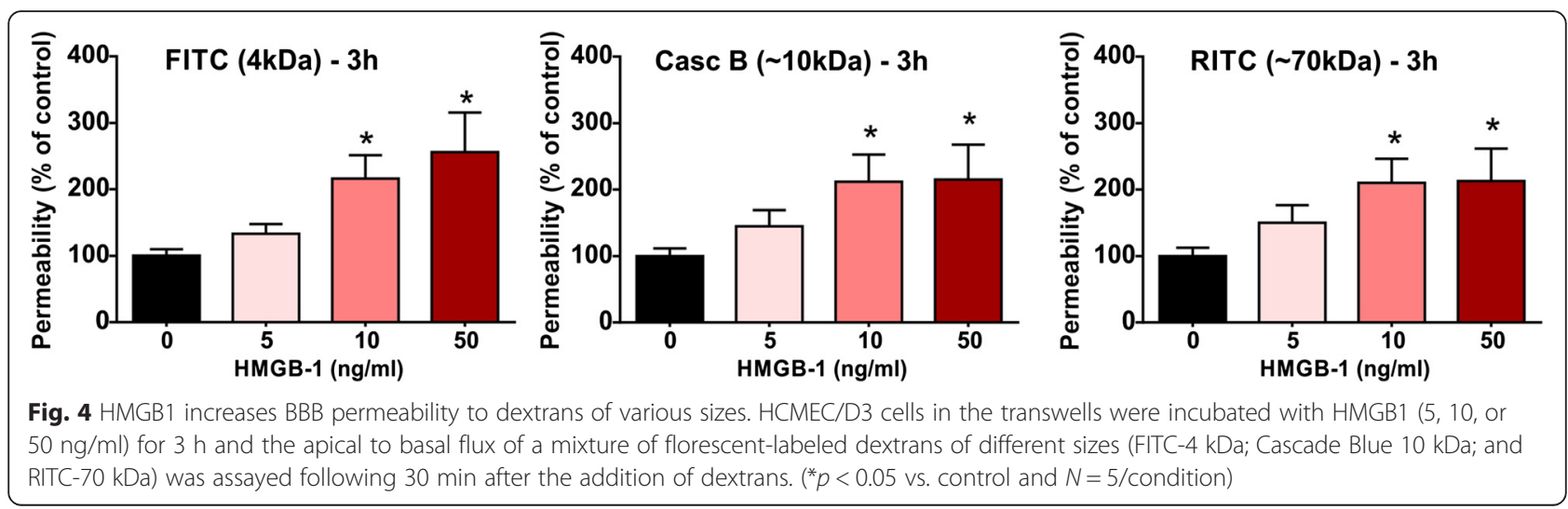


aggregated, TMa was greater in disease $(\mathrm{MCI}+\mathrm{AD})$ than aged-matched controls (see Fig. 2).

As with other vascular barriers $[17,18]$, human alpha thrombin significantly compromised $\mathrm{BBB}$ integrity in vitro at nanomolar concentrations (Fig. 3b), orders of magnitude less than the circulating prothrombin levels in the blood [38]. In an attempt to explain the thrombin's effect on brain edema, Guan and colleagues injected thrombin stereotactically into the rat caudate nuclei and found a marked extravasation of Evans Blue dye, suggesting BBB disruption [42]. These authors also demonstrated that exposure to thrombin upregulated the endothelial expression of matrix metalloproteinase2 possibly through the activation of protease-activated receptor 1 (PAR1). In similar direct experiments using different vascular barrier models, Garcia's group and others showed that thrombin mediated barrier disruption, indeed, via a PAR1 mechanism [17, 18, 43]. Although, we have no current direct evidence that PAR1 mediated the BBB disruption effects of thrombin, the nanomolar concentrations are in agreement with mediation via PAR1. However, our preliminary experiments suggested that the BBB disruptive effects of thrombin are a direct measure of its proteolytic activity, since recombinant hirudin, a specific thrombin inhibitor, inhibited the effects of thrombin (data not shown). Further indicating thrombin was likely interacting with a protease-activated receptor (PAR) on the EC surface, an rTM fragment that binds both thrombin and PC (TMD23) also blocked thrombin's effects on BBB permeability (not shown).

The proinflammatory DAMP, HMGB1, dramatically enhanced BBB permeability to various molecular weight dextrans in primary HBMEC or hCMEC/D3 cultures, following incubation for 3 or $6 \mathrm{~h}$. These BBB changes including the significant downregulation of ZO-1 expression at intercellular (TJs) clearly mirror what has been observed in vivo in animal studies that suggest the presence of amyloid beta is a factor in the "breach" of the BBB [44]. In experimental animals and cell cultures, beta amyloid proteins induce matrix metalloproteinase-9 (MMP-9) [45]. Transgenic mutant mice with mutant human amyloid protein show disruption of the BBB [46]. Furthermore, transgenic mice with apoE4 demonstrate BBB breakdown by activating the proinflammatory cyclophilin A-MMP-9 pathway in the brain pericytes, which, in turn, results in degradation of the BBB TJs and basal lamina (BL) proteins [47]. Mice overexpressing $\mathrm{A} \beta$-precursor protein have pericyte loss, elevated brain $A \beta 40$ and $A \beta 42$ levels accelerating amyloid angiopathy, and cerebral amyloidosis [48].

Others have also shown that BBB dysfunction in rats, whether due to experimental stroke or traumatic brain injury (TBI), can be prevented by a neutralizing antibody to HMGB1 $[49,50]$. Outside the cell, oxidized HMGB1 is known to ligate three different pattern recognition receptors all expressed on the surface of these ECs (as illustrated in Fig. 5). These include TLR2, TLR4, and RAGE [51, 52], and each can bind other ligands, such as $\mathrm{A} \beta$, contributing to vascular complications of other diseases [53]. Both TLR and RAGE signaling leads to downstream NFkB activation with subsequent increase in the expression/release of tumor necrosis factor (TNF), thus ensuring that the inflammatory signal is maintained and amplified [54].

Over the last 15 years, the concept that the BBB might function both as source and target of proinflammatory factors is supported by numerous studies showing that the cerebral microcirculation is in an "activated proinflammatory" stage in neurodegenerative diseases such as $\mathrm{AD}[15,55]$ and is a target for various proinflammatory factors. PAUSE Circulating $A \beta$ is a proinflammatory cytokine-induced DAMP that binds to RAGE and TLR2 [56]. Like S100 $\beta$ and HMGB1, A $\beta$ can exacerbate proinflammatory cytokine production such as TNF and interleukin-1 beta (IL-1 $\beta$ ); however, in innate immunity, TNF dominates. When HMGB1 binds to RAGE and/or TLRs on microvascular ECs, it releases TNF and other cytokines [37], making the BBB also a source of these proinflammatory factors, and the axis in $\mathrm{AD}$ has attracted considerable attention [56]. Soluble RAGE or sRAGE, sometimes referred to as endogenous secretory RAGE, which can be produced either by splicing or proteolytic shedding, appears to play the role of a "decoy" to tie up RAGE ligands, such as S100/calgranulin family members, HMGB1, or A $\beta$ [39]. The S100B/RAGE axis has been of considerable interest in $\mathrm{AD}$, but earlier reports suggested that lower sRAGE levels characterized $\mathrm{AD}$ [39]. Interestingly, we found significant correlation between $A \beta$ and HMGB1 or sRAGE (Fig. 1). Furthermore, the levels of HMGB1 in serum were in the range of rHMGB1 used in the BBB permeability experiments (Figs. 3a and 4), suggesting a continuum from normal through MCI to AD may exist. We did not find an indication that S100B might be useful, although a trend of significance in the MCI due to $\mathrm{AD}$ cohort was found (Fig. 1).

Just in the last decade, it has become increasingly appreciated that inflammation and coagulation are linked evolutionary defense systems [41], a fact that is slowly becoming recognized in the CNS as well. TBI and ischemic and hemorrhagic stroke are all characterized by increased levels of intraparenchymal thrombin and HMGB1 as well as evidence of BBB dysfunction [42, 49, 50]. In the brain, low concentrations of thrombin act through its principal receptor, PAR1, to induce neuroprotection [57]. In contrast, at higher concentrations, thrombin causes brain damage [58], where we showed 


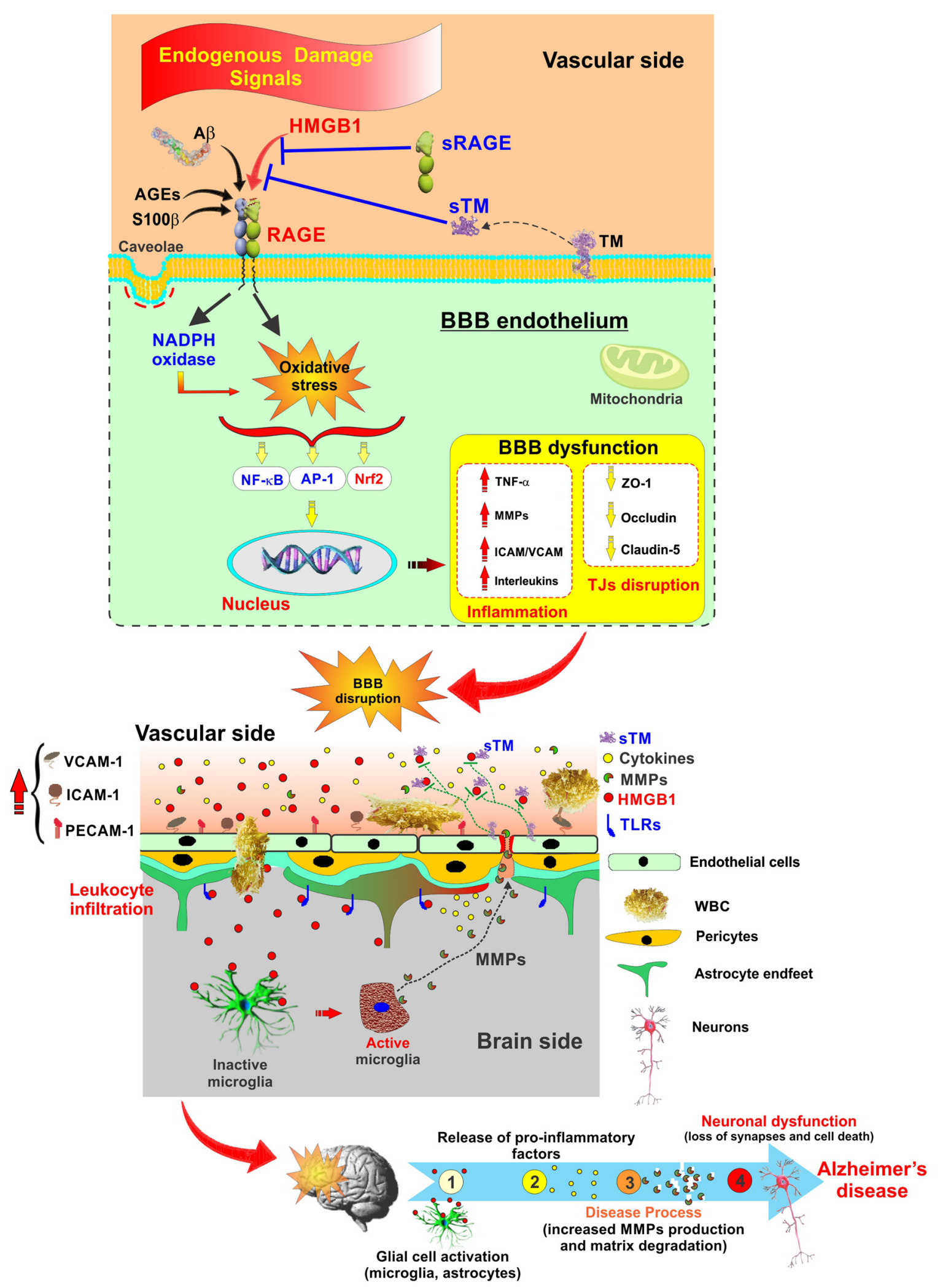

Fig. 5 (See legend on next page.) 
(See figure on previous page.)

Fig. 5 Schematic illustration of proinflammatory AB, DAMPs (HMGB1/S100B), and SRAGE acting at the BBB endothelium. Representation of BBB as source and target of neuroinflammation in AD. Endogenous damage molecules, DAMPs as well as AB, activate respective receptors to ramp up neuroinflammation. Soluble receptors such as SRAGE and sTM can neutralize some of these, and some may function as distinct biomarkers in progression of neurodegeneration in $A D$

that it appears to act via PAR4 [59-61]. Through PAR activation, thrombin directly affects the activity of multiple cell types and regulates a variety of biological functions, including inflammation, leukocyte migration, and vascular permeability [61]. Relevant to this and our current results, all PARs are expressed in microvascular ECs [62], and PAR1, at least, is expressed in ECs of the BBB [63].

Also attesting to evolutionary linkage, direct relationships also exist between thrombin and HMGB1. HMGB1 is involved in a number of systemic vascular diseases [64], is increased in stroke [65], and found around amyloid plaques and NFTs in AD brains [66], where thrombin and prothrombin accumulate as well [67]. Both HMGB1 and thrombin are released in various neurologic conditions, and HMGB1 promotes coagulation [68]. Taken together, these observations raise the possibility that HMGB1 and thrombin also participate during innate immune neuroinflammatory situations such as in $\mathrm{AD}$ and which contribute to $\mathrm{BBB}$ dysfunction and $\mathrm{WBC}$ transendothelial migration.

Finally, we have envisioned our present data in corroboration with previous findings schematically in Fig. 5. This shows that increased levels of danger/damage-associated, proinflammatory molecules (the alarmin DAMPs, HMGB1 and S100B, as well as A $\beta$ ) feature prominently in the $\mathrm{AD}$ continuum from normal aging through $\mathrm{MCI}$ by acting on TLRs and RAGE to induce neuroinflammatory signaling at the $\mathrm{BBB}$ endothelium. This results in increased expression of the transcription factor NFkB causing increased TNF and IL-1 $\beta$ (other interleukins), ROS, and adhesion molecules along with degradation of tight junction (TJ) proteins such as ZO-1. Both endogenous as well as exogenous means exist to combat this imbalance including the proteolytic release of sRAGE and sTM, both of which selectively bind up HMGB1 (possibly other alarmins) preventing engagement of RAGE or TLRs. This also allows for the development of novel therapeutic targets that act like sRAGE and sTM, in a manner less burdensome and potentially with fewer side effects than by administering neutralizing antibodies to HMGB1, such as recently used by others in rat models of stroke, TBI, and Parkinson's disease $[49,50,69]$.

\section{Conclusions}

An increased understanding of the role of HMGB1 and thrombin in BBB dysfunction as well as activation and transendothelial migration of WBCs contributing to neuroinflammation in $\mathrm{AD}$ may allow for the discovery of novel therapeutic targets and treatment strategies. Not only might these facilitate treatment to halt progression in $\mathrm{AD}$ but these might also aid in means to detect the conversion from $\mathrm{MCI}$ to pre-clinical $\mathrm{AD}$, as well as in other neurological disorders that display BBB dysfunction.

\section{Acknowledgements}

We thank Dr. KY Kim (KUMC) for the preliminary in vitro BBB experiments and Dr. Michael Bustin (NIH) for the anti-HMGB1 antibody.

\section{Funding}

This work was supported by NIH/NIDA R01-DA029121-01A1 and the Alternative Research Development Foundation grants received by LC. Some studies were partially funded by the Kansas Bioscience Authority (BWF). The KU ADC human study was supported by NIH P30 AG035982 to SRH.

\section{Availability of data and materials}

All raw data used in this manuscript will be made available upon request.

\section{Authors' contributions}

BWF contributed to the concept, experimental design, conductance, data analysis, and interpretation including manuscript drafting, revision, and approval; RKS contributed to the conductance, data collection, and analysis in addition to drafting and revising the manuscript; PVD contributed toward the experimental design, execution, and data analysis; LC contributed to experimental design, data analysis, manuscript writing and revision, as well

as supporting this study. All authors read and approved the final manuscript.

\section{Competing interests}

BWF is the founder and president of pHLOGISTIX LLC.; PVD is the head of the Clinical Research Department at Diagnostica Stago, France; RS and LC have no competing interests.

\section{Consent for publication}

Not applicable.

\section{Ethics approval and consent to participate}

Enrollment into the KU ADC clinical cohort was performed using a protocol and consent form/process that was approved by the University of Kansas Medical Center's (KUMC) Human Subject's Committee (protocol \# 11132).

\section{Author details}

${ }^{1}$ pHLOGISTIX LLC, 4220 Shawnee Mission Parkway, Fairway, KS 66205, USA. ${ }^{2}$ Department of Neurology, University of Kansas Medical Center, 3901 Rainbow Blvd, Kansas City, KS 66160, USA. ${ }^{3}$ Department of Pharmaceutical Sciences, Texas Tech University Health Sciences Center, 1300 S. Coulter Street, Amarillo, TX 79106, USA. ${ }^{4}$ Clinical Research Department, R\&D,

Diagnostica Stago, Gennevilliers, France.

Received: 1 April 2016 Accepted: 17 August 2016

Published online: 24 August 2016

\section{References}

1. Cummings JL, Morstorf T, Zhong K. Alzheimer's disease drug-development pipeline: few candidates, frequent failures. Alzheimers Res Ther. 2014;6:37.

2. Bloom GS. Amyloid-beta and tau: the trigger and bullet in Alzheimer disease pathogenesis. JAMA Neurol. 2014;71:505-8.

3. Hsiao K, Chapman P, Nilsen S, Eckman C, Harigaya Y, Younkin S, Yang F, Cole G. Correlative memory deficits, Abeta elevation, and amyloid plaques in transgenic mice. Science. 1996;274:99-102. 
4. Deane R, Bell RD, Sagare A, Zlokovic BV. Clearance of amyloid-beta peptide across the blood-brain barrier: implication for therapies in Alzheimer's disease. CNS Neurol Disord: Drug Targets. 2009;8:16-30.

5. Park L, Zhou P, Koizumi K, El Jamal S, Previti ML, Van Nostrand WE, Carlson G, ladecola C. Brain and circulating levels of Abeta1-40 differentially contribute to vasomotor dysfunction in the mouse brain. Stroke. 2013;44:198-204

6. Tuppo EE, Arias HR. The role of inflammation in Alzheimer's disease. Int J Biochem Cell Biol. 2005;37:289-305

7. Hensley K. Neuroinflammation in Alzheimer's disease: mechanisms, pathologic consequences, and potential for therapeutic manipulation. J Alzheimers Dis. 2010;21:1-14.

8. Mhatre M, Floyd RA, Hensley K. Oxidative stress and neuroinflammation in Alzheimer's disease and amyotrophic lateral sclerosis: common links and potential therapeutic targets. J Alzheimers Dis. 2004;6:147-57.

9. Ferretti MT, Cuello AC. Does a pro-inflammatory process precede Alzheimer's disease and mild cognitive impairment? Curr Alzheimer Res. 2011:8:164-74.

10. Zlokovic BV. Neurovascular pathways to neurodegeneration in Alzheimer's disease and other disorders. Nat Rev Neurosci. 2011;12:723-38.

11. Muoio V, Persson PB, Sendeski MM. The neurovascular unit-concept review. Acta Physiol (Oxf). 2014;210:790-8.

12. Keaney J, Campbell M. The dynamic blood-brain barrier. FEBS J. 2015;282:4067-79

13. Redwine L, Mills PJ, Sada M, Dimsdale J, Patterson T, Grant I. Differential immune cell chemotaxis responses to acute psychological stress in Alzheimer caregivers compared to non-caregiver controls. Psychosom Med. 2004;66:770-5

14. Zaghi J, Goldenson B, Inayathullah M, Lossinsky AS, Masoumi A, Avagyan H, Mahanian M, Bernas M, Weinand M, Rosenthal MJ, et al. Alzheimer disease macrophages shuttle amyloid-beta from neurons to vessels, contributing to amyloid angiopathy. Acta Neuropathol. 2009;117:111-24.

15. Grammas P, Martinez J, Miller B. Cerebral microvascular endothelium and the pathogenesis of neurodegenerative diseases. Expert Rev Mol Med. 2011;13:e19.

16. Heppner FL, Ransohoff RM, Becher B. Immune attack: the role of inflammation in Alzheimer disease. Nat Rev Neurosci. 2015;16:358-72.

17. Birukova AA, Birukov KG, Smurova K, Adyshev D, Kaibuchi K, Alieva I, Garcia $J G$, Verin AD. Novel role of microtubules in thrombin-induced endothelial barrier dysfunction. FASEB J. 2004;18:1879-90.

18. Garcia JG. Concepts in microvascular endothelial barrier regulation in health and disease. Microvasc Res. 2009;77:1-3.

19. Wolfson RK, Chiang ET, Garcia JG. HMGB1 induces human lung endothelial cell cytoskeletal rearrangement and barrier disruption. Microvasc Res. 2011:81:189-97.

20. Nawaz MI, Mohammad G. Role of high-mobility group box-1 protein in disruption of vascular barriers and regulation of leukocyte-endothelial interactions. J Recept Signal Transduct Res. 2015:35:340-5.

21. Dumitriu IE, Baruah P, Manfredi AA, Bianchi ME, Rovere-Querini P. HMGB1: guiding immunity from within. Trends Immunol. 2005;26:381-7.

22. Wang $H$, Bloom $O$, Zhang $M$, Vishnubhakat JM, Ombrellino $M$, Che J, Frazier

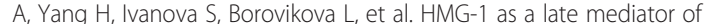
endotoxin lethality in mice. Science. 1999;285:248-51.

23. Schiraldi M, Raucci A, Munoz LM, Livoti E, Celona B, Venereau E, Apuzzo T, De Marchis F, Pedotti M, Bachi A, et al. HMGB1 promotes recruitment of inflammatory cells to damaged tissues by forming a complex with CXCL12 and signaling via CXCR4. J Exp Med. 2012;209:551-63.

24. Venereau E, Schiraldi M, Uguccioni M, Bianchi ME. HMGB1 and leukocyte migration during trauma and sterile inflammation. Mol Immunol. 2013:55:76-82

25. Mazarati A, Maroso M, lori V, Vezzani A, Carli M. High-mobility group box-1 impairs memory in mice through both toll-like receptor 4 and receptor for advanced glycation end products. Exp Neurol. 2011;232:143-8.

26. Iwashyna TJ, Ely EW, Smith DM, Langa KM. Long-term cognitive impairment and functional disability among survivors of severe sepsis. JAMA. 2010;304: 1787-94.

27. Frank-Cannon TC, Alto LT, McAlpine FE, Tansey MG. Does neuroinflammation fan the flame in neurodegenerative diseases? Mol Neurodegener. 2009;4:47

28. Clark IA, Vissel B. Amyloid beta: one of three danger-associated molecules that are secondary inducers of the proinflammatory cytokines that mediate Alzheimer's disease. Br J Pharmacol. 2015;172:3714-27.
29. McKhann GM, Knopman DS, Chertkow H, Hyman BT, Jack Jr CR, Kawas CH, Klunk WE, Koroshetz WJ, Manly JJ, Mayeux R, et al. The diagnosis of dementia due to Alzheimer's disease: recommendations from the National Institute on Aging-Alzheimer's Association workgroups on diagnostic guidelines for Alzheimer's disease. Alzheimers Dement. 2011;7:263-9.

30. Albert MS, DeKosky ST, Dickson D, Dubois B, Feldman HH, Fox NC, Gamst A, Holtzman DM, Jagust WJ, Petersen RC, et al. The diagnosis of mild cognitive impairment due to Alzheimer's disease: recommendations from the National Institute on Aging-Alzheimer's Association workgroups on diagnostic guidelines for Alzheimer's disease. Alzheimers Dement. 2011;7:270-9.

31. Sajja RK, Green KN, Cucullo L. Altered Nrf2 signaling mediates hypoglycemia-induced blood-brain barrier endothelial dysfunction in vitro. PLoS One. 2015;10:e0122358

32. Dhillon NK, Peng F, Bokhari S, Callen S, Shin SH, Zhu X, Kim KJ, Buch SJ. Cocaine-mediated alteration in tight junction protein expression and modulation of CCL2/CCR2 axis across the blood-brain barrier: implications for HIV-dementia. J Neuroimmune Pharmacol. 2008;3:52-6.

33. Van Dreden P, Rousseau A, Savoure A, Lenormand B, Fontaine S, Vasse M. Plasma thrombomodulin activity, tissue factor activity and high levels of circulating procoagulant phospholipid as prognostic factors for acute myocardial infarction. Blood Coagul Fibrinolysis. 2009;20:635-41.

34. Santilli F, Vazzana N, Bucciarelli LG, Davi G. Soluble forms of RAGE in human diseases: clinical and therapeutical implications. Curr Med Chem. 2009;16:940-52.

35. Yamagishi S, Matsui T. Soluble form of a receptor for advanced glycation end products (sRAGE) as a biomarker. Front Biosci (Elite Ed). 2010;2:1184-95.

36. Festoff BW, Li C, Woodhams B, Lynch S. Soluble thrombomodulin levels in plasma of multiple sclerosis patients and their implication. J Neurol Sci. 2012;323:61-5.

37. Fiuza C, Bustin M, Talwar S, Tropea M, Gerstenberger E, Shelhamer JH, Suffredini AF. Inflammation-promoting activity of HMGB1 on human microvascular endothelial cells. Blood. 2003;101:2652-60.

38. Conway EM. Thrombomodulin and its role in inflammation. Semin Immunopathol. 2012;34:107-25.

39. Leclerc E, Sturchler E, Vetter SW. The S100B/RAGE axis in Alzheimer's disease. Cardiovasc Psychiatry Neurol. 2010;2010:539581.

40. Yavuz BB, Dede DS, Yavuz B, Cankurtaran M, Halil M, Ulger Z, Cankurtaran ES, Aytemir K, Kabakci G, Haznedaroglu IC, Ariogul S. Potential biomarkers for vascular damage in Alzheimer's disease: thrombomodulin and von Willebrand factor. J Nutr Health Aging. 2010;14:439-41.

41. Delvaeye M, Conway EM. Coagulation and innate immune responses: can we view them separately? Blood. 2009:114:2367-74.

42. Guan JX, Sun SG, Cao XB, Chen ZB, Tong ET. Effect of thrombin on blood brain barrier permeability and its mechanism. Chin Med J (Engl). 2004;117:1677-81.

43. Bogatcheva NV, Garcia JG, Verin AD. Molecular mechanisms of thrombininduced endothelial cell permeability. Biochemistry (Mosc). 2002;67:75-84.

44. Carmeliet $P$, De Strooper B. Alzheimer's disease: a breach in the blood-brain barrier. Nature. 2012:485:451-2.

45. Hartz AM, Bauer B, Soldner EL, Wolf A, Boy S, Backhaus R, Mihaljevic I, Bogdahn U, Klunemann HH, Schuierer G, Schlachetzki F. Amyloid-beta contributes to blood-brain barrier leakage in transgenic human amyloid precursor protein mice and in humans with cerebral amyloid angiopathy. Stroke. 2012;43:514-23.

46. Kook SY, Seok Hong H, Moon M, Mook-Jung I. Disruption of blood-brain barrier in Alzheimer disease pathogenesis. Tissue Barriers. 2013;1:e23993.

47. Bell RD, Winkler EA, Singh I, Sagare AP, Deane R, Wu Z, Holtzman DM, Betsholtz C, Armulik A, Sallstrom J, et al. Apolipoprotein E controls cerebrovascular integrity via cyclophilin A. Nature. 2012:485:512-6.

48. Sagare AP, Bell RD, Zhao Z, Ma Q, Winkler EA, Ramanathan A, Zlokovic BV. Pericyte loss influences Alzheimer-like neurodegeneration in mice. Nat Commun. 2013;4:2932.

49. Zhang J, Takahashi HK, Liu K, Wake H, Liu R, Maruo T, Date I, Yoshino T, Ohtsuka A, Mori S, Nishibori M. Anti-high mobility group box-1 monoclonal antibody protects the blood-brain barrier from ischemia-induced disruption in rats. Stroke. 2011:42:1420-8.

50. Okuma Y, Liu K, Wake H, Zhang J, Maruo T, Date I, Yoshino T, Ohtsuka A, Otani N, Tomura S, et al. Anti-high mobility group box-1 antibody therapy for traumatic brain injury. Ann Neurol. 2012;72:373-84.

51. Hori O, Brett J, Slattery T, Cao R, Zhang J, Chen JX, Nagashima M, Lundh ER, Vijay $S$, Nitecki $D$, et al. The receptor for advanced glycation end products 
(RAGE) is a cellular binding site for amphoterin. Mediation of neurite outgrowth and co-expression of rage and amphoterin in the developing nervous system. J Biol Chem. 1995;270:25752-61.

52. van Beijnum JR, Buurman WA, Griffioen AW. Convergence and amplification of toll-like receptor (TLR) and receptor for advanced glycation end products (RAGE) signaling pathways via high mobility group B1 (HMGB1). Angiogenesis. 2008;11:91-9.

53. Bierhaus A, Humpert PM, Stern DM, Arnold B, Nawroth PP. Advanced glycation end product receptor-mediated cellular dysfunction. Ann N Y Acad Sci. 2005;1043:676-80.

54. Bierhaus A, Humpert PM, Morcos M, Wendt T, Chavakis T, Arnold B, Stern DM, Nawroth PP. Understanding RAGE, the receptor for advanced glycation end products. J Mol Med (Berl). 2005;83:876-86.

55. Rochfort KD, Cummins PM. The blood-brain barrier endothelium: a target for pro-inflammatory cytokines. Biochem Soc Trans. 2015;43:702-6.

56. Liu S, Liu Y, Hao W, Wolf L, Kiliaan AJ, Penke B, Rube CE, Walter J, Heneka MT, Hartmann T, et al. TLR2 is a primary receptor for Alzheimer's amyloid beta peptide to trigger neuroinflammatory activation. J Immunol. 2012;188:1098-107.

57. Striggow F, Riek M, Breder J, Henrich-Noack P, Reymann KG, Reiser G. The protease thrombin is an endogenous mediator of hippocampal neuroprotection against ischemia at low concentrations but causes degeneration at high concentrations. Proc Natl Acad Sci U S A. 2000;97:2264-9.

58. Xi G, Reiser G, Keep RF. The role of thrombin and thrombin receptors in ischemic, hemorrhagic and traumatic brain injury: deleterious or protective? J Neurochem. 2003;84:3-9.

59. Suo Z, Wu M, Citron BA, Gao C, Festoff BW. Persistent protease-activated receptor 4 signaling mediates thrombin-induced microglial activation. J Biol Chem. 2003;278:31177-83.

60. Suo Z, Wu M, Citron BA, Palazzo RE, Festoff BW. Rapid tau aggregation and delayed hippocampal neuronal death induced by persistent thrombin signaling. J Biol Chem. 2003;278:37681-9.

61. Suo Z, Citron BA, Festoff BW. Thrombin: a potential proinflammatory mediator in neurotrauma and neurodegenerative disorders. Curr Drug Targets Inflamm Allergy. 2004;3:105-14.

62. Hirano K. The roles of proteinase-activated receptors in the vascular physiology and pathophysiology. Arterioscler, Thromb, Vasc Biol. 2007:27:27-36

63. Bartha K, Domotor E, Lanza F, Adam-Vizi V, Machovich R. Identification of thrombin receptors in rat brain capillary endothelial cells. J Cereb Blood Flow Metab. 2000;20:175-82.

64. de Souza AW, Westra J, Limburg PC, Bijl M, Kallenberg CG. HMGB1 in vascular diseases: its role in vascular inflammation and atherosclerosis. Autoimmun Rev. 2012;11:909-17.

65. Fang P, Schachner M, Shen YQ. HMGB1 in development and diseases of the central nervous system. Mol Neurobiol. 2012;45:499-506.

66. Takata K, Takada T, Ito A, Asai M, Tawa M, Saito Y, Ashihara E, Tomimoto H, Kitamura Y, Shimohama S. Microglial amyloid-beta1-40 phagocytosis dysfunction is caused by high-mobility group box protein-1: implications for the pathological progression of Alzheimer's disease. Int J Alzheimers Dis. 2012:2012:685739.

67. Arai T, Miklossy J, Klegeris A, Guo JP, McGeer PL. Thrombin and prothrombin are expressed by neurons and glial cells and accumulate in neurofibrillary tangles in Alzheimer disease brain. J Neuropathol Exp Neurol. 2006;65:19-25.

68. Ito T, Kawahara K, Nakamura T, Yamada S, Nakamura T, Abeyama K, Hashiguchi T, Maruyama I. High-mobility group box 1 protein promotes development of microvascular thrombosis in rats. J Thromb Haemost. 2007;5:109-16

69. Sasaki T, Liu K, Agari T, Yasuhara T, Morimoto J, Okazaki M, Takeuchi H, Toyoshima A, Sasada S, Shinko A, et al. Anti-high mobility group box 1 antibody exerts neuroprotection in a rat model of Parkinson's disease. Exp Neurol. 2016;275(Pt 1):220-31.

\section{Submit your next manuscript to BioMed Central and we will help you at every step:}

- We accept pre-submission inquiries

- Our selector tool helps you to find the most relevant journal

- We provide round the clock customer support

- Convenient online submission

- Thorough peer review

- Inclusion in PubMed and all major indexing services

- Maximum visibility for your research

Submit your manuscript at www.biomedcentral.com/submit
Biomed Central 